\title{
Influence de la hauteur du massif filtrant sur l'élimination des polluants organiques dans un filtre à sable à alimentation intermittente
}

\author{
Béatrice Assamoi AMA-CAUPHYS*, Amos Kouadio KOUAMÉ, Aman MESSOU et \\ Lacina COULIBALY
}

Laboratoire d'Environnement et de Biologie Aquatique, UFR-Sciences et Gestion de l'Environnement, 02 BP 801 Abidjan 02, Côte d'Ivoire.

*Auteur correspondant; E-mail : ama_beatrice@yahoo.fr, Tel : (00225) 0102174470

\author{
Received: 23-03-2021 $\quad$ Accepted: 27-09-2021 $\quad$ Published: 30-10-2021
}

\section{RÉSUMÉ}

La pollution des eaux de surface par les eaux usées urbaines est une préoccupation environnementale majeure. Cette étude a consisté à développer des filtres à sable intermittent en série en forme de U et à étudier l'influence de la hauteur et de la granulométrie du lit filtrant sur l'enlèvement des polluants organiques des eaux usées. Ainsi, deux hauteurs de lits filtrants $(60 \mathrm{~cm})$ et $(70 \mathrm{~cm})$ ont été utilisés. Les schistes grossiers, schistes moyens et le sable de lagune ont constitué le massif filtrant. Les résultats montrent que les rendements obtenus avec les filtres $70 \mathrm{~cm}$ de lit filtrant $\left(\mathrm{FH}_{70}\right)$ sont relativement plus élevés (MES : 77,16\%, DCO : 89,75\% et $\mathrm{DBO}_{5}$ : $92,96 \%)$. Cependant, les schistes moyens et le sable de lagune permettent d'obtenir une bonne réduction des MES, de la DCO et $\mathrm{DBO}_{5}$ dans les filtrats par rapport aux schistes grossiers. Les analyses du profil des polluants organiques dans le filtre de $70 \mathrm{~cm}$ de massif filtrant ont montré une diminution des teneurs de ces polluants de la surface au fond du filtre et elles restent élevées dans la couche de 0 à $20 \mathrm{~cm}$. Les résultats obtenus montrent que les filtres $\mathrm{FH}_{70}$ contenant des schistes moyens et du sable de lagune constituent une technologie efficace d'épuration des eaux usées.

(C) 2021 International Formulae Group. All rights reserved.

Mots clés : filtre à sable intermittent, eaux usées urbaines, massif filtrant, matière organique, schiste, canalisation d'eau pluviale.

\section{Effect of sand depth on the removal of organic pollutants in an intermittent sand filter}

\begin{abstract}
Pollution of surface waters by urban wastewater is a major environmental concern. This study was conducted to develop intermittent sand filters in the shape of a "U" for organic pollutants treatment. In this study, the effect of both two sand depth $(60 \mathrm{~cm}$ and $70 \mathrm{~cm})$ and the filter media grain size on the removal of organic pollutants was studied. The coarsest shale medium (Sch1), mean shale (Sch2) and white lagoon sand constituting the filter media. The results show that the filter of $70 \mathrm{~cm}$ of sand $\left(\mathrm{FH}_{70}\right)$ was the most efficient (TSS: $77.16 \%$, COD: $89.75 \%$ and BOD5: $92.96 \%$ ). However, the media grain size which removed optimum MES, DCO and DBO5 was observed to be mean shale and the white lagoon. Concerning the organic matter concentration in filter
\end{abstract}


medium of filter $70 \mathrm{~cm}$ of sand, they decrease with depth and the sand layer from 0 to $20 \mathrm{~cm}$ show the highest concentration. The results obtained show that the filter of $70 \mathrm{~cm}$ of mean shale and with lagoon sand is an efficient technology for treating treat urban wastewater.

(C) 2021 International Formulae Group. All rights reserved.

Keywords: Intermittent sand filter, urban wastewater, media filter, organic matter, shale, stormwater channel.

\section{INTRODUCTION}

Les eaux usées urbaines constituent la plus grande source de pollution des milieux récepteurs. Dans les pays en développement, un grand nombre de cours d'eau restent très pollués par le déversement des eaux usées urbaines en quantités de plus en plus importantes à cause de l'explosion démographique, de l'urbanisation et de l'industrialisation (Fouad et al., 2014). En Côte d'Ivoire et dans le district d'Abidjan en particulier, $24000 \mathrm{~m}^{3}$ d'eaux usées produites par jour sont rejetés sans traitement adéquat (Koanda, 2006). De plus Ama et al. (2009) ont démontré que les canalisations d'eau pluviale d'Abidjan sont anthropisées par les déchets industriels, domestiques et artisanaux. Par conséquent, les eaux usées non traitées se déversent dans la lagune Ébrié. Cette situation est due à une insuffisance des stations d'épuration des eaux usées municipales du district d'Abidjan. Ces effluents rejetés sont caractérisés de plus en plus par de fortes charges organiques, de fortes demandes chimiques en oxygène, d'une biotoxicité accrue à l'égard des bactéries et des concentrations élevées en nutriments (Aslan, 2001 ; Alvarado-Lassman et al., 2008 ; Agler et al., 2010). Par ailleurs, une forte présence de matière organique en suspension dans le milieu aquatique cause de nombreux problèmes entre autres, la coloration, les odeurs et de la consommation de chlore. Sa fraction biodégradable provoque la décroissance bactérienne et la consommation d'oxygène dissous de ces eaux, entrainant l'eutrophisation et la mort des poissons (Georgantas et Grigoropoulou, 2006 ; Dovonou et al., 2011). Afin d'éliminer au mieux ces polluants organiques responsables de la dégradation de la qualité de l'eau dans le temps, divers procédés ont été expérimentés: procédés physique (Achak et al., 2008), thermique (Le Cloirec,
2004 ; Renou, 2006; Colombano, 2010), coagulation et floculation (Moukorab et al., 2018) et les procédés biologiques (Yu et al., 2000 ; Ménoret, 2001 ; Azimi et Zamanzadeh, 2004). Les procédés biologiques sont certes efficaces et économiques pour le traitement de ces eaux, mais ils requièrent souvent des temps de rétentions élevés et l'installation de bassin de grande dimension. Les procédés physicochimiques quant à eux requièrent des temps de rétention relativement courts (Massé et Masse, 2000), mais utilisent des concentrations importantes de sels métalliques augmentant ainsi le volume des boues générées (Meçabih et al., 2006; Tatangelo, 2006) et par conséquent, le coût du traitement. Les procédés thermiques génèrent de fortes dépenses énergétiques et peuvent nécessiter la mise en place d'un dispositif de traitement des fumées émises (Krou, 2010). Au vu de ces difficultés, il apparaît urgent de rechercher des méthodes alternatives simples et robustes de traitement de la matière organique. L'enlèvement des matières organiques des effluents par adsorption sur des argiles en particulier, la bentonite a été investiguée (Meçabih et al., 2006 ; ACHAK et al., 2009). Pour ces procédés, les capacités d'adsorption tendent à diminuer avec le temps, à cause d'une diminution des sites actifs libres. Quant à la bentonite, les capacités épuratoires sont optimisées lorsqu'elle est associée aux sels de fer et d'aluminium. Toutefois, le filtre à sable à alimentation intermittente en série en forme de $\mathrm{U}$ et garnie de schiste concassé pourrait constituer une alternative intéressante pour l'élimination des matières organiques des eaux usées. Ces filtres sous forme de colonne en série, agissent comme des bassins de sédimentation de grande surface et à long temps de séjour, ce qui permet de minimiser leur colmatage. De plus, le schiste qui est un très bon absorbant est susceptible de libérer du 
fer et de l'aluminium capable en présence d'eau, de générer des groupements hydroxydes pouvant être impliqués dans les réactions d'adsorption de la matière organique (Deschamps et al., 2006). Cette méthode ne génère pas des boues chimiques à traiter en plus d'être moins coûteuse et les matériaux utilisés dans la conception sont très accessibles. Malgré ces avantages, peu d'études ont été réalisées sur les filtres à sable alimentation intermittente dans les pays africains particulièrement en Côte d'Ivoire pour appréhender les paramètres de dimensionnement, dont la hauteur du lit filtrant sur la qualité physique et chimique des eaux usées. C'est dans ce cadre que cette étude a été initiée. L'objectif principal de cette étude était d'étudier l'effet de la hauteur du lit filtrant sur l'élimination des polluants organiques des eaux usées urbaines issues des collecteurs d'eaux pluviales dans un filtre à sable Intermittent.

\section{MATÉRIEL ET MÉTHODES Dispositif expérimental}

Un filtre pilote à alimentation intermittente (Figure 1) a été conçu et utilisé dans cette étude. Le support des filtres est en PVC $(11 \mathrm{~cm}$ de diamètre et de hauteur 105 à $115 \mathrm{~cm})$. Ce dispositif est composé de trois compartiments $\mathrm{C} 1, \mathrm{C} 2$ et $\mathrm{C} 3$ de forme $\mathrm{U}$ respectivement montés en série. Chaque compartiment est constitué d'une colonne filtrante et d'une colonne de sédimentation. $\mathrm{La}$ colonne filtrante est garnie du bas vers le haut de $15 \mathrm{~cm}$ de gravier, d'un géotextile et de matériaux filtrants. Le montage est fait de sorte que la granulométrie du massif filtrant diminue du premier compartiment au troisième pour chaque réacteur. La première et la seconde colonne filtrante ont été garnies par du schiste, avec des schistes grossiers à granulométrie uniforme $(\mathrm{m}=3650 \mu \mathrm{m}, \mathrm{CU}=1,66)$ dans la première colonne filtrante du compartiment $\mathrm{C} 1$, schiste moyen à granulométrie variée $(\mathrm{m}=$ 1416.67 $\mu \mathrm{m}, \mathrm{CU}=5$ ) dans la seconde colonne filtrante du compartiment (C2) et de sable moyen à granulométrie variée $(\mathrm{m}=466,67$, $\mathrm{CU}=2,5)$ dans la troisième colonne du dernier compartiment. Au total, deux filtres ont été réalisés. Pour étudier l'effet de la hauteur du massif filtrant sur le fonctionnement du filtre, deux hauteurs de massif ont été utilisées. L'étude a consisté à augmenter de $10 \mathrm{~cm} \mathrm{la}$ hauteur de massif filtrant à partir de $50 \mathrm{~cm}$ utilisés lors des essais de traitement de Ama (2013). Ainsi, l'un des filtres a été rempli de 60 $\mathrm{cm}$ de massif filtrant et l'autre filtre de $70 \mathrm{~cm}$. Tous les filtres ont été alimentés au débit de 7,5 $\mathrm{L} / \mathrm{J}$.

\section{Préparation des massifs filtrants}

Le schiste et le sable utilisés (Figure 2) ont été prélevés respectivement sur le site de Lomo-Nord (Toumodi, Côte d'Ivoire) et d'une carrière de sable de la lagune Ébrié. Les schistes ont été concassés pour obtenir deux types de granulométrie après tamisage avec des tamis de mailles $5 \mathrm{~mm}$ et $2 \mathrm{~mm}$. Les schistes sont capables de relarguer du fer et de l'aluminium et composés de $55,43 \%$ de $\mathrm{SiO}_{2}$, $15,46 \%$ de $\mathrm{Al}_{2} \mathrm{O}_{3}$, et $9,21 \%$ de $\mathrm{Fe}_{2} \mathrm{O}_{3}$. Le Sable est constitué à $99 \%$ de grain de quartz et d'environ $1 \%$ de muscovite (micas) et de détritus calcaire provenant des coquilles et des déchets des organismes marins.

\section{Échantillonnage des eaux usées, des filtrats et des substrats}

Les eaux usées utilisées ont été prélevées dans le collecteur d'eau pluviale du boulevard Latrille situé dans la Commune de Cocody, Abidjan (Coordonnées géographiques : $\mathrm{N} 05^{\circ} 20^{\prime} 59,8^{\prime \prime}$ : E $004^{\circ} 00^{\prime}$ 09,6 '). Les populations qui y résident raccordent de façon illicite leurs systèmes d'évacuations des eaux usées à cause des coûts élevés du raccordement au système d'égout (Ouattara, 2005). Ces eaux ont été stockées après prélèvement dans des bidons plastiques de vingt litres (20L). Ensuite, les échantillons ont été transportés sur le site expérimental pour alimenter les filtres.

L'alimentation des filtres consiste à appliquer des eaux usées sur le massif du compartiment $\mathrm{C} 1$ du système de réacteurs. Ensuite, sous l'effet de la gravité, l'eau s'infiltre dans les autres compartiments en traversant successivement le massif et la colonne de sédimentation des compartiments 
C2 et C3. Un échantillon d'eaux usées brutes et le filtrat (eau traitée) du réacteur ont été prélevés séparément dans des flacons de $0,5 \mathrm{~L}$ une fois par semaine et conservés à $4^{\circ} \mathrm{C}$ jusqu'à l'analyse. Le filtrat a été prélevé dans les colonnes de sédimentation et à la sortie du réacteur. Les paramètres mesurés sont le $\mathrm{pH}$, la conductivité, les matières en suspension (MES), la demande biologique en oxygène pendant 5 jours $\left(\mathrm{DBO}_{5}\right)$ et la demande chimique en oxygène (DCO).

Pour évaluer la quantité de matière organique dans le substrat (massif filtrant) des réacteurs, l'analyse du profil de concentration de la matière organique a été effectuée dans le filtre ayant fourni le meilleur rendement d'élimination. Ainsi, des échantillons ont été prélevés dans les trois compartiments du filtre de hauteur $70 \mathrm{~cm}$ de massif par carottage du substrat du réacteur à l'aide d'une tarière en polychlorure de vinyle (PVC) de diamètre 40 $\mathrm{mm}$ entre 0 et $60 \mathrm{~cm}$. Trois (3) couches de substrat ont été considérées suivant le profil vertical du réacteur : une couche supérieure de 0 à $20 \mathrm{~cm}$ de profondeur, une couche intermédiaire de 20 à $40 \mathrm{~cm}$ et une couche inférieure de 40 à $60 \mathrm{~cm}$. L'échantillonnage a été réalisé à la fin de l'essai de traitement et les échantillons ont été conservés dans des sachets plastiques pour analyse.

\section{Analyses physico-chimiques}

Les matières en suspension (MES) ont été déterminées par la méthode AFNOR NF T90-105. Ces matières en suspension (MES) ont été mesurées sur des échantillons décantés pendant une demi-heure. Un volume (V) de 10 $\mathrm{ml}$ du surnageant a été prélevé et porté dans un creuset pré-pesé (M1). L'ensemble creuset et échantillon a été séché à $105^{\circ} \mathrm{C}$ pendant $1 \mathrm{~h} 30$ min dans une étuve. Ensuite, il a été retiré de l'étuve et laissé à la température ambiante dans un dessiccateur puis pesé (M2). Les MES ont été calculées suivant la relation (1) :

$$
\operatorname{MES}(\mathrm{g} / \mathrm{L})=\frac{M 2-M 1}{10} \times 1000
$$

La détermination de la DC $U$ repose sur une oxydation par un excès de dichromate de potassium dosé par le sulfate de fer et d'ammonium conformément à la norme AFNOR T90101.
Quant à la détermination de la demande biologique en oxygène pendant 5 jours $\left(\mathrm{DBO}_{5}\right)$, elle a visé à reconstituer en laboratoire les phénomènes de dégradation qui ont lieu dans le milieu naturel selon la méthode AFNOR (1975).

La conductivité a été mesurée à l'aide d'un multi-paramètre de marque INOLAB 7310 .

Sur chacun des échantillons de substrat prélevés, le carbone organique a été dosé selon la norme NF ISO 10694 (AFNOR, 1995), à partir de laquelle la matière organique correspondante a été déterminée. Cette méthode est basée sur l'oxydation du carbone organique par le dichromate de potassium (K2CrO7) en milieu acide.

\section{Rendement épuratoire}

Les rendements épuratoires des paramètres chimiques à la sortie de différentes colonnes de sédimentation de chaque compartiment sont calculés suivant les relations (2), (3) et (4) :

$$
\begin{gathered}
R(C 1)=\frac{X E B-X 1}{X E B} \times 100 \\
R(C 2)=\frac{X 1-X 2}{X 1} \times 100 \\
R(C 3)=\frac{X 2-X 3}{X 2} \times 100
\end{gathered}
$$

Avec :

$\mathrm{XEB}=$ Concentration du paramètre considéré dans l'eau usée appliquée,

$\mathrm{X} 1, \mathrm{X} 2$ et $\mathrm{X} 3$ = Concentrations du paramètre considéré dans le filtrat des compartiments $\mathrm{C} 1, \mathrm{C} 2$ et $\mathrm{C} 3$ respectivement.

\section{Analyses statistiques}

Une analyse de variance ANOVA à un facteur et ANOVA de kruskal Wallis a été utilisée pour tester la variation entre les paramètres physico-chimiques de l'eau brute, des filtrats et des rendements épuratoires entre les différents réacteurs. Elle a été suivie par le test post hoc $\mathrm{t}$ pour comparer les valeurs moyennes de ces paramètres entre les réacteurs pris deux à deux. Les tests ont été réalisés à l'aide du logiciel STATISTICA version 7.1 à un seuil de significativité $\mathrm{p}<0,05$. 

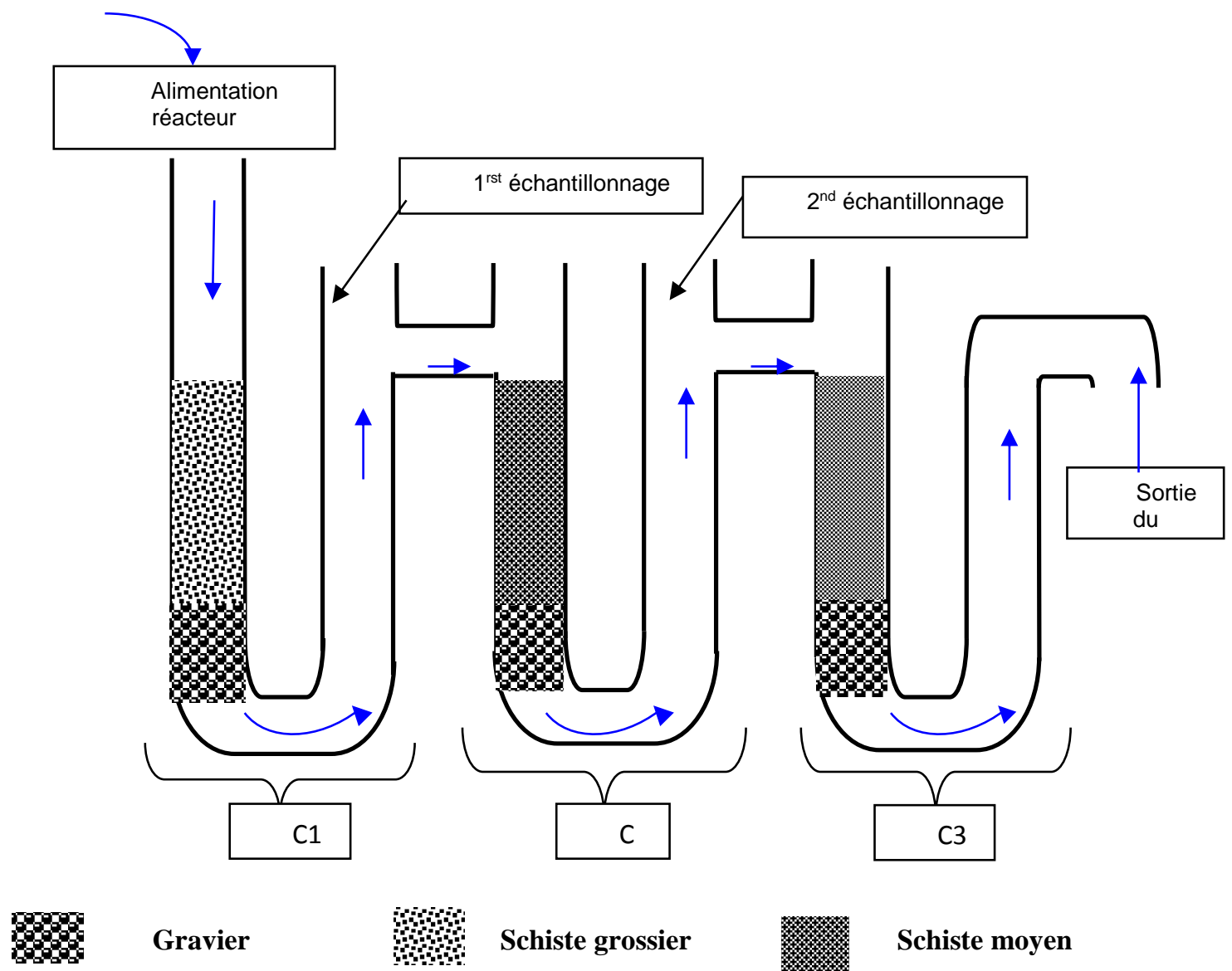

\section{Gravier}

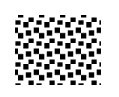

Schiste grossier

\section{Schiste moyen}

Sable blanc de lagune

$\longrightarrow$ Trajet de l'écoulement

Figure 1 : Configuration du réacteur ; $\mathrm{C} 1=$ Compartiment $1 ; \mathrm{C} 2=$ Compartiment $2 ; \mathrm{C} 3=$ Compartiment 3 .
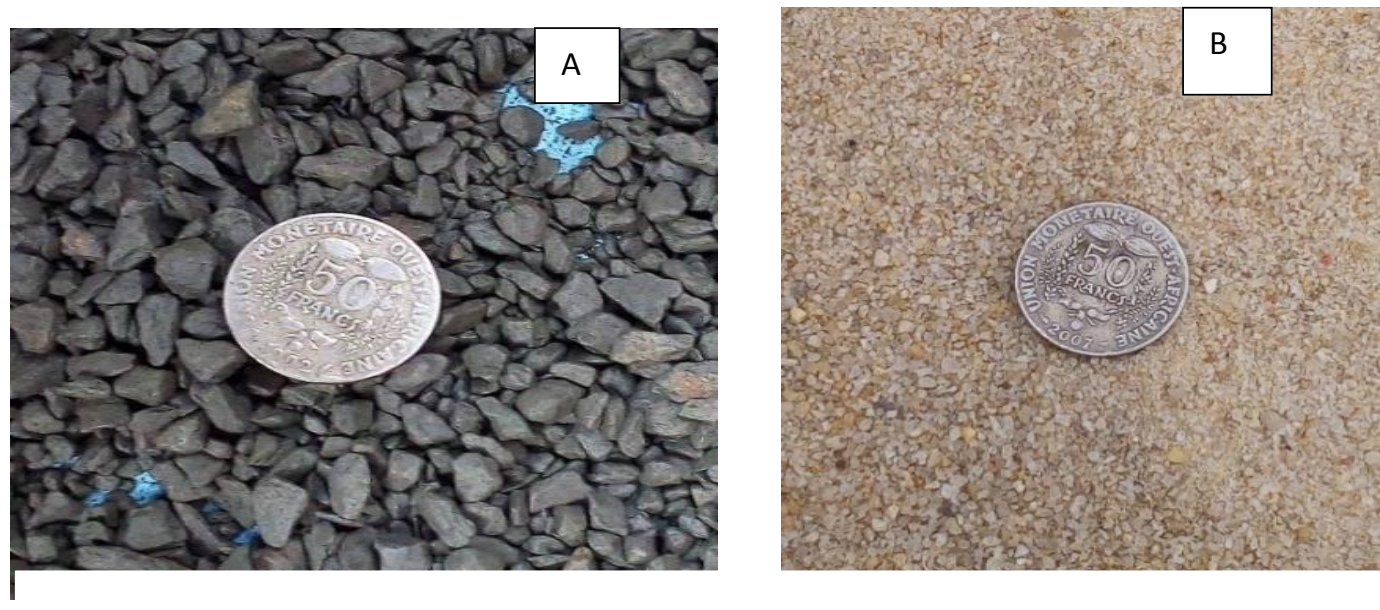

Figure 2 : Massif filtrants utilisés : schiste concassé (A) et sable de lagune (B). 


\section{RÉSULTATS \\ Performance épuratoire $p H$}

La Figure 3 présente l'évolution du $\mathrm{pH}$ de l'eau usée brute (EB) et des différents filtrats. Dans l'ensemble, le $\mathrm{pH}$ des filtrats augmente avec la hauteur du massif filtrant. Les valeurs moyennes varient entre 7,26 et 7,55 pour le filtre de $60 \mathrm{~cm}$ de lit filtrant $\left(\mathrm{FH}_{60}\right)$ et varient entre 7,21 et 7,64 pour le filtre de $70 \mathrm{~cm}$ de lit filtrant $\left(\mathrm{FH}_{70}\right)$. Les résultats montrent que le compartiment $(\mathrm{C} 2)$ garni de schiste moyen à granulométrie variée et celui garni de sable moyen à granulométrie variée $(\mathrm{C} 3)$ ont les $\mathrm{pH}$ les plus élevés. Le $\mathrm{pH}$ mesuré dans les filtrats du compartiment $\mathrm{C} 3$ est supérieur à celui de l'eau brute (EB) $(\mathrm{pH}=7,41)$. Les valeurs de $\mathrm{pH}$ de l'eau brute et celles des filtrats issus des filtres $\mathrm{FH}_{60}$ et $\mathrm{FH}_{70}$ sont significativement différentes (ANOVA Kruskal-Wallis : $\mathrm{p}<0,05)$, quel que soit le type de massif filtrant.

\section{Conductivité électrique (EC)}

La conductivité des filtrats passe de 609,19 à $619,47 \mu \mathrm{s} . \mathrm{cm}^{-1}$ en moyennes lorsque la hauteur du massif filtrant augmente de 60 à $70 \mathrm{~cm}$ (Figure 4). En considérant la granulométrie des massifs filtrants, les résultats montrent que le compartiment garni de massifs grossiers uniformes (C1) a la conductivité électrique la plus élevée. En effet, les valeurs moyennes sont de $654,14 \mu \mathrm{s} . \mathrm{cm}^{-1}, 603,57$ $\mu \mathrm{s} . \mathrm{cm}^{-1}$ et $569,85 \mu \mathrm{s} . \mathrm{cm}^{-1}$ respectivement pour les compartiments $\mathrm{C} 1, \mathrm{C} 2$ et $\mathrm{C} 3$ du filtre $\mathrm{FH}_{60}$ (Figure 4A) et de $665,57 \mu \mathrm{s} . \mathrm{cm}^{-1}$ pour $\mathrm{C} 1$, $615,57 \mu \mathrm{s} . \mathrm{cm}^{-1}$ pour $\mathrm{C} 2$ et $577,28 \mu \mathrm{s} . \mathrm{cm}^{-1}$ pour $\mathrm{C} 3 \mathrm{du}$ filtre $\mathrm{FH}_{70}$ (Figure 4B). On ne note aucune différence significative (Test $t, P>$ $0,05)$ entre la conductivité des filtrats du filtre $\mathrm{FH}_{60}$ et $\mathrm{FH}_{70}$.

\section{Matières en suspension (MES)}

La Figure 5 présente l'influence de la hauteur du massif filtrant sur le rendement épuratoire de MES. La concentration de MES des filtrats est inférieure à celle de l'eau brute (EB) quelle que soit la hauteur du massif utilisé. La concentration moyenne de MES pour les eaux brutes est de $678,14 \mathrm{mg}$. $\mathrm{L}^{-1}$ contre respectivement $207,57 \mathrm{mg}$. $\mathrm{L}^{-1}$ et 154,85 mg. $\mathrm{L}^{-1}$ pour les filtrats des filtres $\mathrm{FH}_{60}$ (Figure 5 A) et $\mathrm{FH}_{70}$ (Figure 5B). On obtient un abattement de $69,39 \%$ pour $\mathrm{FH}_{60}$ et $(77,16 \%)$ pour $\mathrm{FH}_{70}$. Par ailleurs, le rendement d'élimination de MES augmente avec la diminution de la granulométrie du massif filtrant (de $\mathrm{C} 1$ à $\mathrm{C} 3$ ). Le pourcentage de réduction de MES à la sortie des colonnes de sédimentation des compartiments $\mathrm{C} 1, \mathrm{C} 2$ et $\mathrm{C} 3$ du $\mathrm{FH}_{60}$ (Figure 5A) est d'environ 25,23\%, $31,81 \%$ et $39,95 \%$ respectivement. Celui à la sortie des colonnes de sédimentation des compartiments $\mathrm{C} 1, \mathrm{C} 2$ et $\mathrm{C} 3$ dans le filtre $\mathrm{FH}_{70}$ (Figure 5B) est de $33,68 \%, 39,19 \%$ et $43,36 \%$ respectivement. Une différence significative (Test $t, \quad p<0,05)$ est notée entre les concentrations de MES de l'eau brute (EB) et celles des filtrats des différents compartiments. Par contre, les concentrations de MES des filtrats ne sont pas significativement différentes (Test $\mathrm{t}, \mathrm{p}>0,05$ ).

\section{Demande chimique en oxygène (DCO)}

La Figure 6 illustre l'évolution de la concentration de la demande chimique en oxygène (DCO) à l'entrée et à la sortie des filtres $\mathrm{FH}_{60}$ et $\mathrm{FH}_{70}$. On constate que la concentration de la DCO des filtrats est inférieure à celle de l'eau brute (EB). La concentration dans l'eau brute est de 289,85 $\mathrm{mg}$. $\mathrm{L}^{-1}$ tandis que celle obtenue dans les filtrats est de $39,42 \mathrm{mg}$. $\mathrm{L}^{-1}$ et $29,71 \mathrm{mg}$. $\mathrm{L}^{-1}$ respectivement pour $\mathrm{FH}_{60}$ et $\mathrm{FH}_{70}$. Ce qui donne un abattement de $86,39 \%$ pour le Filtre $\mathrm{FH}_{60}$ et $89,75 \%$ pour le $\mathrm{FH}_{70}$. En considérant l'effet de la granulométrie des massifs filtrants, le schiste moyen à granulométrie variée (compartiment $\mathrm{C} 2$ ) et le sable moyen à granulométrie variée (compartiment C3) permettent d'obtenir une bonne réduction de la DCO. La concentration moyenne de DCO relevée à la sortie des compartiments $\mathrm{C} 1, \mathrm{C} 2$ et $\mathrm{C} 3 \mathrm{du}$ filtre $\mathrm{FH}_{60}$ est de $181,28 \mathrm{mg}$. $\mathrm{L}^{-1}, 92,86$ mg. $\mathrm{L}^{-1}$ et $39,42 \mathrm{mg}$. $\mathrm{L}^{-1}$ respectivement (Figure 6A) tandis que celle dans le $\mathrm{FH}_{70}$ est de 147,14 mg. $\mathrm{L}^{-1}$ pour $\mathrm{C} 1,64,85 \mathrm{mg}$. $\mathrm{L}^{-1}$ pour $\mathrm{C} 2$ et 29,71 mg. $\mathrm{L}^{-1}$ pour $\mathrm{C} 3$ (Figure $6 \mathrm{~B}$ ). Le pourcentage de réduction de la DCO est $37,45 \%$ dans $\mathrm{C} 1$, $48,77 \%$ dans $\mathrm{C} 2$ et $57,53 \%$ dans $\mathrm{C} 3$ pour le $\mathrm{FH}_{60}$ tandis que pour le $\mathrm{FH}_{70}$, il est de 49,23\%, $55,92 \%$ et $54,18 \%$ respectivement dans $\mathrm{C} 1, \mathrm{C} 2$ et C3. Une différence significative est notée entre les concentrations de DCO de l'EB et 
celles des différents filtrats (test de Kruskall Wallis, $\mathrm{p}<0,05$ ), de même qu'entre $\mathrm{FH}_{60}$ et $\mathrm{FH}_{70}$. Le filtre $\mathrm{H}_{70}$ a enregistré le rendement épuratoire le plus élevé par rapport à celui de $\mathrm{H}_{60}$.

\section{Demande Biochimique en oxygène $\left(\mathrm{DBO}_{5}\right)$}

La Figure 7 montre que la concentration moyenne en $\mathrm{DBO}_{5}$ pour l'eau brute est de 138 $\mathrm{mg} . \mathrm{L}^{-1}$ et elle est supérieure à celle des filtrats. En considérant les filtrats, la concentration de $\mathrm{DBO}_{5}$ dans le $\mathrm{FH}_{70}$ est supérieure à celle de $\mathrm{FH}_{60}$. La concentration relevée à la sortie du filtre $\mathrm{FH}_{60}$ est de $16,14 \mathrm{mg}$. $\mathrm{L}^{-1}$ et de $9,71 \mathrm{mg}$. $\mathrm{L}^{-1}$ à la sortie du filtre FH70. Le pourcentage de réduction est de $88,30 \%$ en moyenne pour le $\mathrm{FH}_{60}$ et $92,96 \%$ pour le $\mathrm{FH}_{70}$. Quant à l'influence de la granulométrie du massif filtrant sur l'élimination de la $\mathrm{DBO}_{5}$, la concentration est élevée dans le compartiment $\mathrm{C} 1$ par rapport à celle des compartiments $\mathrm{C} 2$ et C3. Les valeurs à la sortie des compartiments $\mathrm{C} 1, \mathrm{C} 2$ et $\mathrm{C} 3$ du $\mathrm{FH}_{60}$ varient entre 65 et 115 mg. $\mathrm{L}^{-1}$, entre 25 et $85 \mathrm{mg}$. $\mathrm{L}^{-1}$ et entre 8 et 20 mg. $L^{-1}$ respectivement (Figure 7A). Les pourcentages de réduction sont de $38,50 \%$ pour $\mathrm{C} 1,47,97 \%$ pour $\mathrm{C} 2$ et $63,43 \%$ pour $\mathrm{C} 3$. En revanche, les concentrations de $\mathrm{DBO}_{5}$ à la sortie des compartiments $\mathrm{C} 1, \mathrm{C} 2$ et $\mathrm{C} 3$ du filtre $\mathrm{FH}_{70}$ sont comprises entre 45 et $87 \mathrm{mg}$. $\mathrm{L}^{-1}$, entre 15 et $32 \mathrm{mg}$. $\mathrm{L}^{-1}$ et entre 2 et $15 \mathrm{mg}$. $\mathrm{L}^{-1}$ respectivement (Figure 7B). Ce qui explique qu'en moyenne $51,96 \%$ de la matière biodégradable est éliminée dans le compartiment $\mathrm{C} 1,62,50 \%$ éliminée dans le $\mathrm{C} 2$ et $60,91 \%$ éliminée dans celui de C3. Une différence significative a été enregistrée entre les concentrations de $\mathrm{DBO}_{5}$ de l'eau brute (EB) et des filtrats, et entre celles des filtrats (test $t$, $\mathrm{p}<0,05)$.

\section{Profil de la matière organique dans les substrats du filtre}

La Figure 8 présente la variation de la teneur en matière organique dans les différents horizons de sol du filtre $\mathrm{FH}_{70}$. L'on constate que la teneur en matière organique décroit de la surface vers le fond du filtre. La plus forte teneur a été enregistrée dans l'horizon 0-20 cm et dans le compartiment $\mathrm{C} 1$ garni avec des massifs grossiers uniformes tandis que les plus faibles teneurs ont été relevées dans les horizons $20-40 \mathrm{~cm}$ et $40-60 \mathrm{~cm}$. Les valeurs obtenues varient entre $1653 \mathrm{mg} / \mathrm{kg}$ (C3) et $2116 \mathrm{mg} / \mathrm{kg}(\mathrm{C} 1)$ pour l'horizon $0-20 \mathrm{~cm}$, entre $1295 \mathrm{mg} / \mathrm{kg}$ (C3) et $1754 \mathrm{mg} / \mathrm{kg}$ (C1) pour l'horizon $20-40 \mathrm{~cm}$ et entre 734 et $859 \mathrm{mg} / \mathrm{kg}$ pour l'horizon $40-60 \mathrm{~cm}$. Les concentrations moyennes en matière organique obtenue dans les substrats $\mathrm{du}$ filtre ne sont pas significativement différentes (ANOVA : $\mathrm{p}>$ $0,05)$.

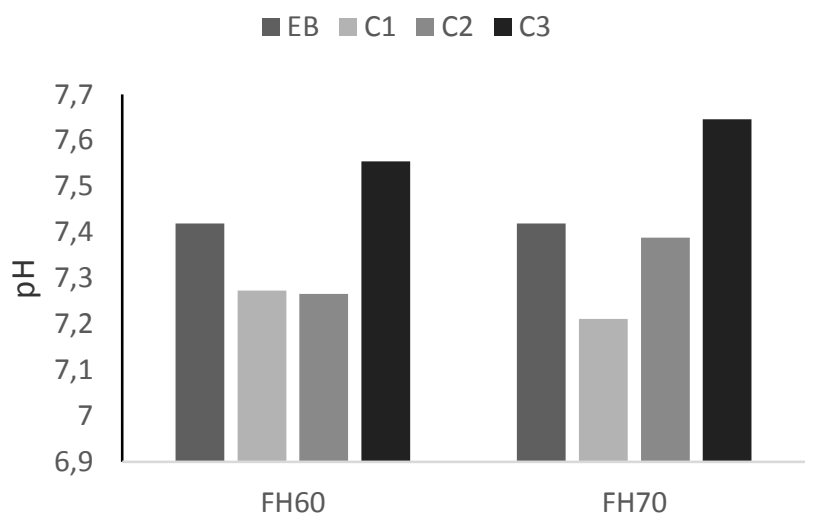

Figure 3 : Influence de la hauteur et de la granulométrie du massif filtrant sur l'évolution du pH de l'eau brute et des filtrats des différents filtres ; $\mathrm{EB}=$ eau brute $; \mathrm{FH}_{60}=$ filtre de $60 \mathrm{~cm}$ de massif filtrant $; \mathrm{FH}_{70}=$ filtre de $70 \mathrm{~cm}$ de massif filtrant. 

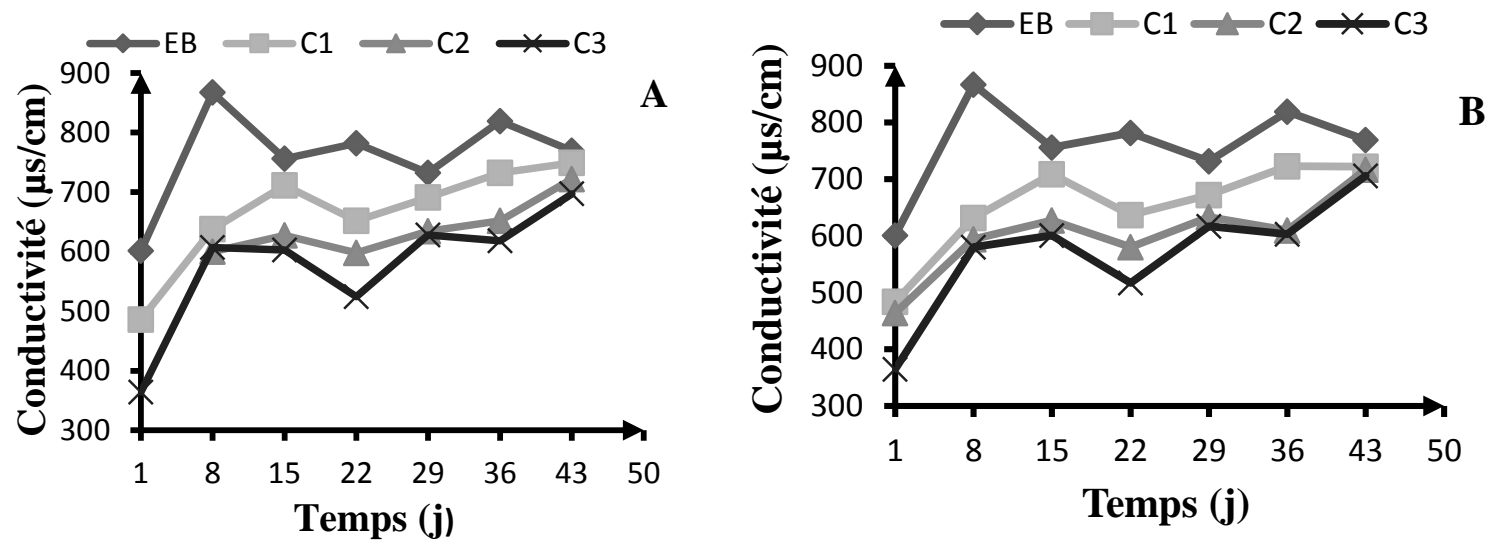

Figure 4 : Évolution de la conductivité de l'eau brute (EB) et des filtrats des différents filtres en fonction du temps ; $\mathrm{A}=$ filtre de $60 \mathrm{~cm}$ de massif filtrant $\left(\mathrm{FH}_{60}\right) ; \mathrm{B}=$ filtre de $70 \mathrm{~cm}$ de
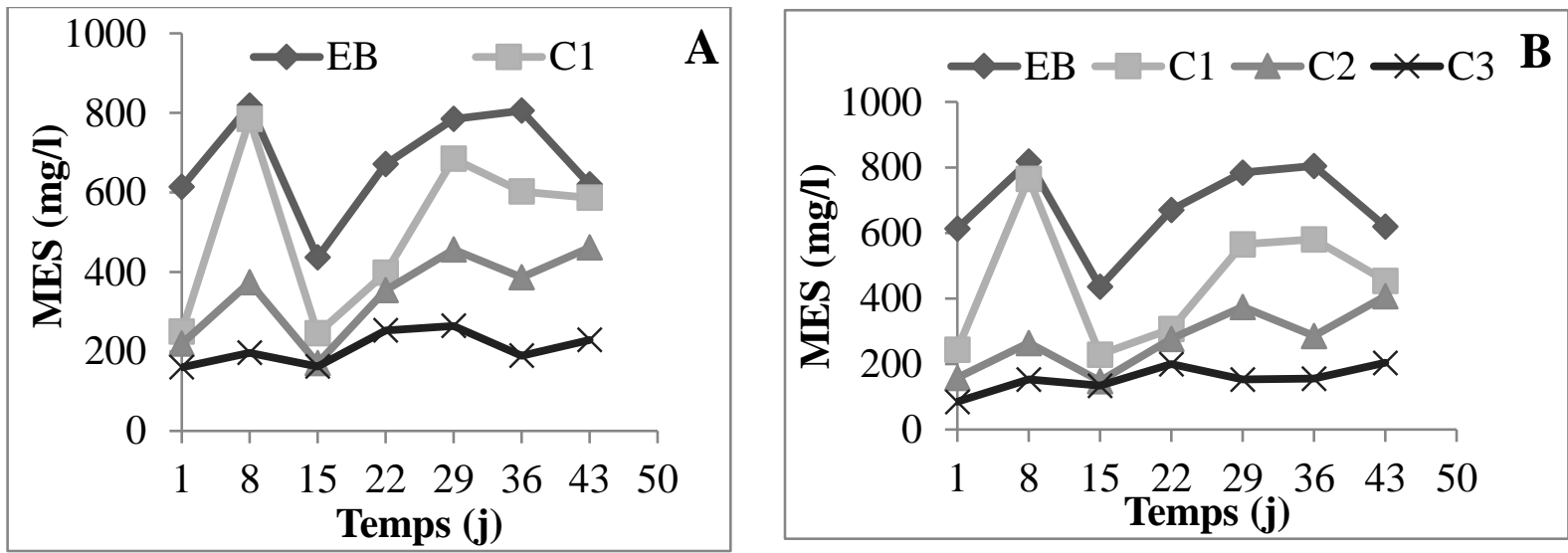

Figure 5 : Évolution des MES de l'eau brute (EB) et des filtrats des différents filtres en fonction du temps ; $\mathrm{A}=$ filtre de $60 \mathrm{~cm}$ de massif filtrant $\left(\mathrm{FH}_{60}\right) ; \mathrm{B}=$ filtre de hauteur $70 \mathrm{~cm}$ de massif filtrant $\left(\mathrm{FH}_{70}\right)$.
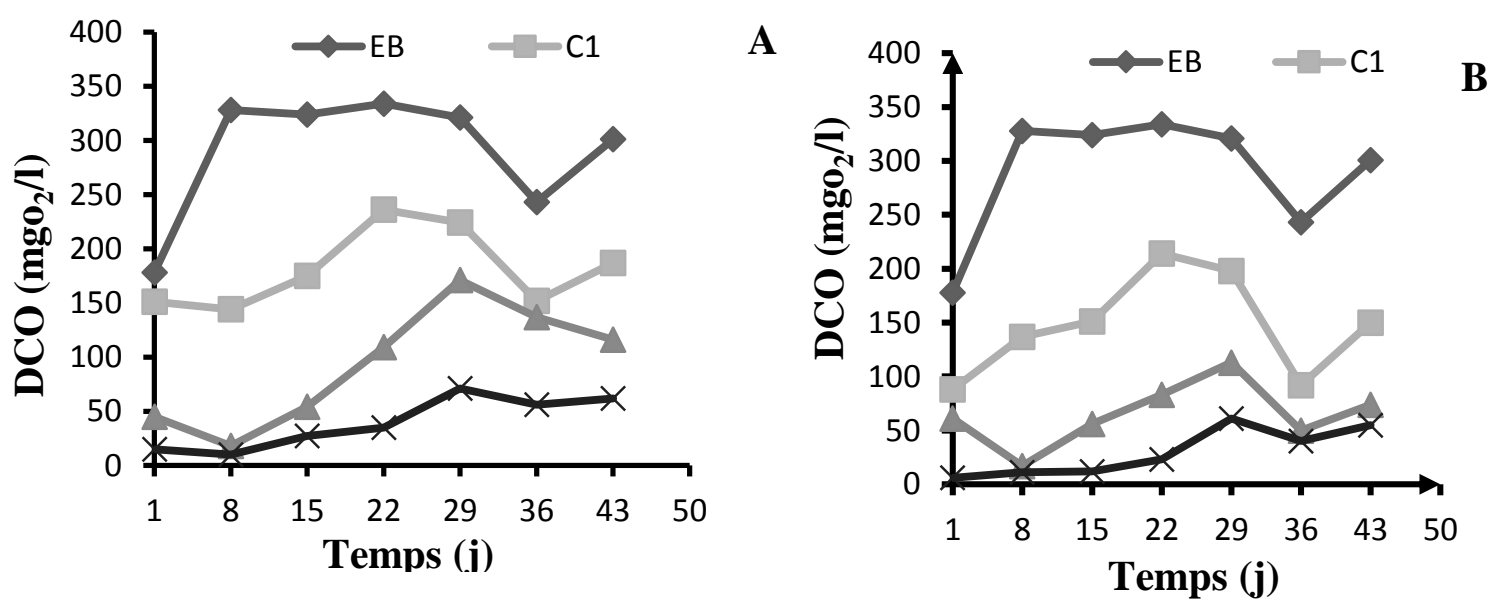

Figure 6 : Évolution de la DCO de l'eau brute (EB) et des filtrats des différents filtres en fonction du temps ; $\mathrm{EB}=$ eau brute $; \mathrm{A}=$ filtre de $60 \mathrm{~cm}$ de massif filtrant $\left(\mathrm{FH}_{60}\right) ; \mathrm{B}=$ filtre de $70 \mathrm{~cm}$ de massif filtrant $\left(\mathrm{FH}_{70}\right)$. 


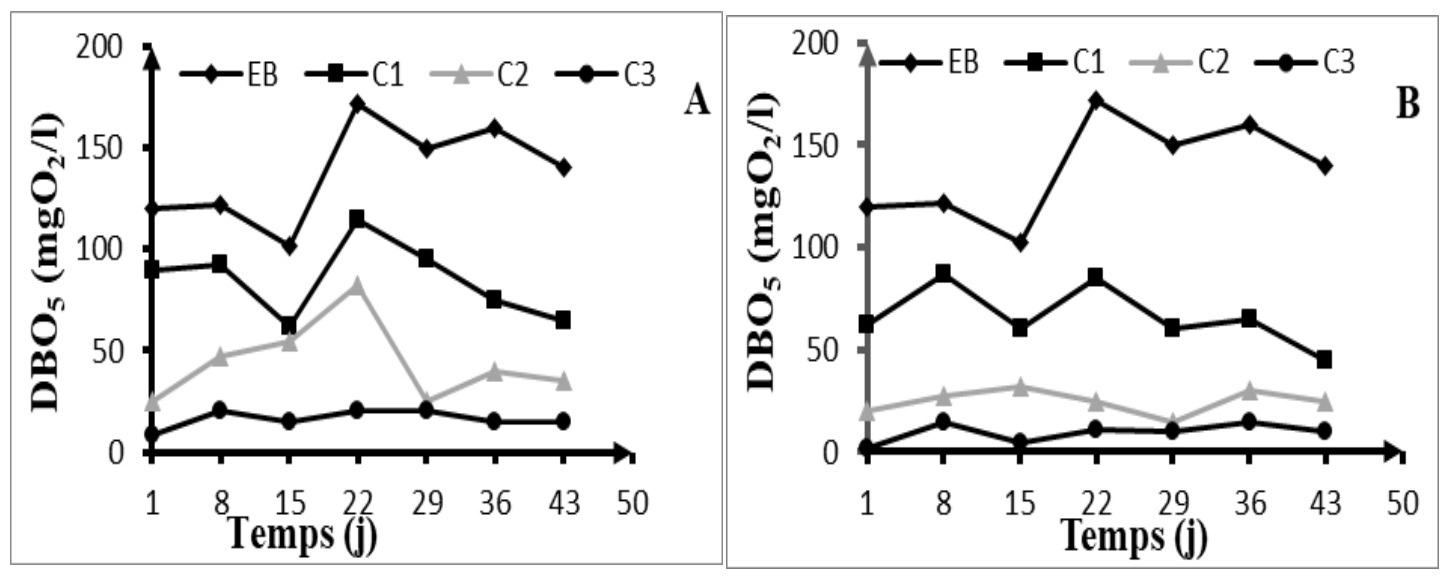

Figure 7 : Évolution de la demande biochimique en oxygène $\left(\mathrm{DBO}_{5}\right)$ en fonction du temps; $\mathrm{EB}=$ eau brute ; $\mathrm{A}=$ filtre de $60 \mathrm{~cm}$ de massif filtrant $\left(\mathrm{FH}_{60}\right) ; \mathrm{B}=$ filtre de $70 \mathrm{~cm}$ de massif filtrant $\left(\mathrm{FH}_{70}\right)$.

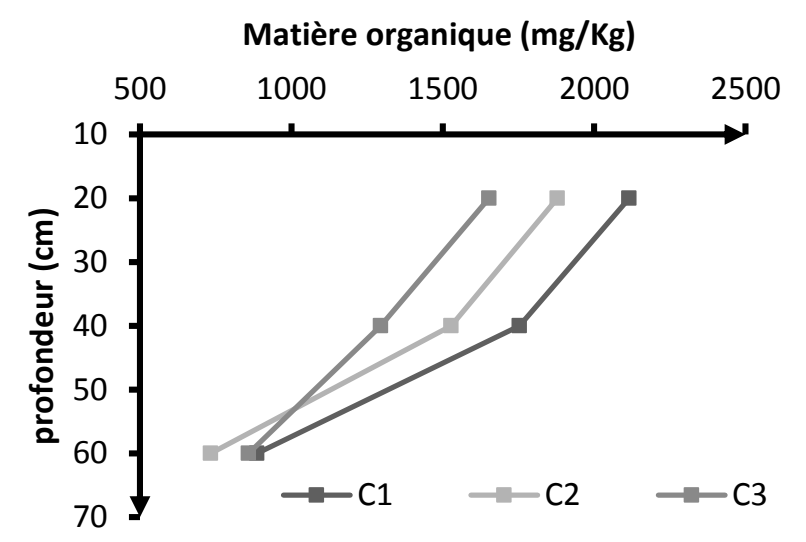

Figure 8 : Variation de la concentration de la matière organique dans les couches de substrat du filtre de 70 $\mathrm{cm}$ de massif filtrant.

\section{DISCUSSION}

L'étude a mis en évidence l'influence de la hauteur du massif et de sa granulométrie sur la capacité épuratoire des filtres à sable à alimentation intermittente montés en série. Concernant le $\mathrm{pH}$, celui des eaux usées brutes varie entre 6.9 et 7.41. Ces valeurs sont celles couramment obtenues dans le cas des eaux usées domestiques (Salghi, 2007), ce qui confirme que les eaux utilisées pour les traitements ont une origine purement domestique. On a observé une augmentation du $\mathrm{pH}$ des filtrats lorsque la hauteur du lit filtrant passe de 60 à $70 \mathrm{~cm}$. Cette tendance à la basification $\mathrm{du} \mathrm{pH}$ des filtrats pourrait s'expliquer par les effets combinés du caractère alcalin du schiste ainsi que la diminution du transfert d'oxygène dans les massifs filtrants des filtres. En effet, l'augmentation de la hauteur du lit filtrant entraine une faible aération du filtre, ce qui réduit les processus de nitrification et d'oxydation de la matière organique au cours desquels le milieu devient acide (Mosely, 2001 ; Tchobanaglous et al., 2003). Des résultats similaires ont été observés par certains auteurs qui les ont expliqués par le même phénomène (Mangoua-Allali et Coulibaly, 2015 ; Ouattara et Coulibaly, 2019). 
Par ailleurs, cette augmentation pourrait s'expliquer par la dissociation des oxydes de fer contenus dans les schistes, ce qui neutraliserait les ions $\mathrm{H}^{+}$des eaux (De Haas et al., 2001). Pour ce qui est de l'influence de la granulométrie, l'élévation du $\mathrm{pH}$ dans les filtrats des massifs fins (compartiment C3) s'expliquerait par la mauvaise oxygénation de ce massif due à la réduction des pores du lit filtrant, ce qui limiterait la décomposition aérobie de la matière organique (Mosely, 2001). C'est d'ailleurs ce qui explique la diminution de la conductivité dans les filtrats des compartiments $\mathrm{C} 3$. Ces résultats sont similaires à ceux obtenus par El Haouti et al. (2016), qui ont trouvé une augmentation du $\mathrm{pH}$ et une diminution de la conductivité des eaux épurées par le sable titanifère tamisé, pendant leurs travaux de recherche sur la filtration sur sable. La totalité des valeurs est conforme aux normes ivoiriennes relatives aux rejets $(6,5<\mathrm{pH}<8,5)$ et les eaux peuvent intégrer le milieu naturel. (Ministère de l'Environnement, des Eaux et forêts, 2008).

Quant aux MES, la concentration de MES baisse lorsque la hauteur du massif filtrant augmente de $60 \mathrm{~cm}$ à $70 \mathrm{~cm}$. Cette élimination serait dépendante de la hauteur du massif filtrant. En effet, selon Alvarez et al. (2007), Healy et al. (2007) et Hericher (2012), plus le massif filtrant est haut, mieux les particules sont retenues progressivement et en grand nombre par le massif. Par ailleurs, ces faibles concentrations de MES observées dans les filtrats s'expliqueraient par la formation des flocs lors de l'hydrolyse du fer et de l'aluminium. Selon Monette et al. (2000), ces sels métalliques $\left(\mathrm{Al}^{3+}\right.$ et $\left.\mathrm{Fe}^{3+}\right)$ contenus dans le schiste neutralisent et déstabilisent les particules colloïdales pour permettre la formation de flocs. Ces flocs sont ensuite piégés par le massif ou déposés par gravité dans les colonnes de sédimentation. Cependant, les valeurs élevées de MES dans les eaux brutes pourraient provenir de la présence de déchets particulaires et colloïdaux dans le collecteur. Ces résultats sont conformes à ceux de Bayoko (2015) qui a montré que les eaux résiduaires $\mathrm{du}$ grand collecteur $\mathrm{du}$ boulevard Latrille avaient une turbidité élevée (turbidité >30 UTN). En outre, les concentrations élevées de MES obtenues dans le compartiment $\mathrm{Cl}$ (schiste grossier) pourraient résulter du lessivage de particules de très petite taille dans la colonne de sédimentation (Van Kam, 2005).

Concernant à la DCO, les valeurs élevées dans les eaux brutes sont principalement dues aux activités domestiques pratiquées dans les environs du collecteur d'eau pluviale. En effet, ces activités rejettent des eaux usées et des déchets solides. La présence de ces activités confirme les résultats de Effebi et al. (2017). Pour eux les matières organiques mesurées dans la lagune Aghien sont les résultats de la baignade, du lavage de la vaisselle, des restes d'aliments, des déversements et de l'utilisation d'engrais chimiques à proximité de la lagune. Par ailleurs, la rétention de la matière particulaire et l'oxydation biologique des matières organiques dissoutes dans les eaux usées par les lits filtrants seraient à la base de la diminution de la concentration de DCO constatée dans les filtrats des réacteurs. Le taux élevé d'élimination de la DCO par le filtre de hauteur $70 \mathrm{~cm}$ est lié à une rétention importante des matières oxydables. Ceci est vraisemblablement dû à la rétention physique de la matière organique de l'eau usée dans le massif filtrant et l'oxydation de celle-ci par des processus biologiques associés à la flore bactérienne (Bancolé et al., 2003 ; Edwin et al., 2006). En effet, la faible conductivité hydraulique due à la forte hauteur de massif filtrant $(70 \mathrm{~cm})$ de ce filtre fait qu'il y a une infiltration lente de l'eau usée dans le massif; ce qui est favorable à la rétention et à la dégradation de la DCO dans le massif. C'est d'ailleurs cette situation qui entraine la diminution des MES, la DCO et $\mathrm{DBO}_{5}$ dans le compartiment $\mathrm{C} 3$. Ces résultats seraient dus à l'existence des pores de petits diamètres dans ces massifs fins qui permettent la rétention d'un maximum de polluants particulaires. Ces faibles concentrations de MES et de la charge organique observées à la sortie du compartiment C3 (sable moyen) sont en conformité avec celles obtenues par Gueye (2007), Koné (2011) et de Ama et al. (2015). En effet, ces auteurs ont obtenu des rendements 
d'élimination de $\mathrm{DBO}_{5}$ de l'ordre de $99 \%$ lorsqu'ils ont utilisé des filtres à sable de granulométrie fine et $53 \%$ pour les filtres à sable garnis de gravier. En revanche, les concentrations élevées, de MES, DCO et $\mathrm{DBO}_{5}$ observées dans le compartiment $\mathrm{C} 1$ (granulométrie grossière) des filtres s'expliqueraient par la réduction du temps de séjour des eaux usées, le lessivage de la biomasse microbienne des massifs filtrants et de la pénétration en profondeur des matières en suspension (Taghizadeh et al., 2007). En effet, au faible temps de séjour, il ne peut qu'avoir une rétention mécanique de MES et de la matière organique qui a lieu principalement en surface.

Relativement aux fortes concentrations de matière organique observée dans les horizons $0-20 \mathrm{~cm}$, elles pourraient être attribuables à la filtration physique de la matière organique qui est retenue essentiellement dans l'horizon de surface (Van Kam, 2005). Cette observation est similaire à celle de Tanja et Jörg (2006). Les travaux de ces auteurs sur l'utilisation de la biomasse du sol comme indicateur de l'élimination biologique du carbone organique dérivé de l'effluent lors de l'infiltration ont montré que la majorité du carbone organique était retenue dans les 30 premiers centimètres de la zone d'infiltration. Par ailleurs, les faibles concentrations de matière organique enregistrées dans les compartiments $\mathrm{C} 2$ et $\mathrm{C} 3$, pourraient s'expliquer par la taille des grains de massif dans ces compartiments. Les compartiments $\mathrm{C} 2$ et $\mathrm{C} 3$ garnis respectivement de schiste moyen et de sable moyen ont les tailles plus petites que celles du massif dans le compartiment $\mathrm{C} 1$. Ces petites tailles de massif offrent une surface spécifique très grande, ce qui augmente le pouvoir de rétention et la dégradation de la matière organique (Shaimali et Singh, 2001). Des résultats similaires ont été obtenus par Kedi et al. (2021) lors de l'élimination des polluants organiques par des argiles naturelles.

\section{Conclusion}

Le présent travail a permis de développer un système de filtre à sable à alimentation intermittente garni de schiste pour éliminer efficacement la matière organique des eaux usées domestiques provenant des collecteurs d'eau pluviale. Les paramètres physico-chimiques analysés (conductivité, $\mathrm{pH}$, DCO, DBO5 et MES) montrent que la qualité de l'eau traitée serait influencée par la hauteur du massif filtrant et de granulométrie de ces massifs. En effet, le filtre de hauteur $70 \mathrm{~cm}$ donnent globalement un meilleur rendement d'élimination de la charge organique des eaux usées avec un pourcentage de réduction de $77,16 \%$ pour les MES, $89,75 \%$ pour la DCO et $92,96 \%$ pour la $\mathrm{DBO}_{5}$ par rapport à celui de hauteur $60 \mathrm{~cm}$ dont le pourcentage de réduction de MES, DCO et DBO5 est de 69,39\%, 86,39\% et $88,30 \%$ respectivement. Les compartiments $\mathrm{C} 2$ et $\mathrm{C} 3$ garnis respectivement de schiste moyen et sable moyen donnent les meilleurs rendements d'élimination pour tous les paramètres étudiés. Relativement au profil de la concentration de la matière organique, l'on a observé qu'il diminue avec la profondeur.

$\mathrm{Au}$ regard des résultats obtenus, le réacteur garni de schiste à granulométrie moyenne et de hauteur de massif $70 \mathrm{~cm}$ peut contribuer énormément à la réduction de la matière organique des eaux usées domestiques provenant des collecteurs d'eau pluviale.

\section{CONFLIT D'INTÉRÊTS}

Les auteurs déclarent qu'ils n'ont pas de conflits d'intérêts par rapport à cet article.

\section{CONTRIBUTIONS DES AUTEURS}

BAA-C a assuré la conduite de l'expérience, la supervision générale des travaux et la rédaction du manuscrit. AKK a assuré la collecte des données, l'analyse, l'interprétation des données et a participé à la rédaction du manuscrit. $\mathrm{AM}$ a participé à la révision du manuscrit. $\mathrm{LC}$ a participé à la conception du filtre expérimental et à la supervision des travaux.

\section{REMERCIEMENTS}

Nous remercions tous les chercheurs de l'Unité de Recherche en Biotechnologie et Ingénierie pour leurs contributions. 


\section{RÉFÉRENCES}

Achak M, Ouazzani N, Yaacoubi A, Mandi L. 2008. Caractérisation des margines issues d'une huilerie moderne et essais de leur traitement par coagulation floculation par la chaux et le sulfate d'aluminium. Rev. Sci. Eau, 21: 53-57. DOI: https://doi.org/10.7202/017930ar

Achak M., Ouazzani N, Mandi L. 2009. Traitement des margines d'une huilerie moderne par infiltration-percolation sur un filtre à sable. Rev. Sci. Eau, 22: 421433.

DOI: https://doi.org/10.7202/045826ar

AFNOR. 1975. Détermination de la demande biochimique en oxygène $\left(\mathrm{DBO}_{5}\right): \mathrm{NF}$ T90-103.

Agler MT, Aydinkaya Z, Cummings TA, Beers AR, Angenent LT. 2010. Anaerobic digestion of brewery primary sludge to enhance bioenergy generation: A comparison between low-and high-rate solids treatment and different temperatures. Bioresour. Technol., 101(15): $5842-5851 . \quad$ DOI: 10.1016/j.biortech.2010.03.023

Alvarado-Lassman A, Rustrian E, GarciaAlvarado MA, Rodriguez-Jiménez GC, Houbron E. 2008. Brewery wastewater treatment using anaerobic inverse fluidized bed reactors. Bioresour. Technol., 99(8): 3009-3015. DOI: 10.1016/j.biortech.2007.06.022

Ama BA. 2013. Gestions des eaux usées domestiques : caractérisation en réseau et prétraitement à l'aide d'un filtre à sable a alimentation intermittente. Thèse de Doctorat Université Nangui Abrogoua, Abidjan (Côte d'Ivoire), p. 289.

Ama AB, Coulibaly L, Savané I. 2009. Dynamique comparée des nutriments $(\mathrm{N}$, $\mathrm{P}$ ) et des solides dans deux collecteurs d'Eaux pluviales anthropisés par les rejets industriels et domestiques à Abidjan, Côte D'Ivoire. Eur. J. Sci. Res., 25(2) : 280-289.

http://www.eurojournals.com/ejsr.htm

Ama AB, Koné T, Ouattara PJ-M, Coulibaly L, Savané I. 2015. An intermittent sand filter for domestic wastewater treatment: effect of hydraulic loading rate on the kinetic and amylase activity. JCBPS, 5(1): 10101021.

Arslan I. 2001. Treatability of a simulated disperse dye-bath by ferrous iron coagulation ozonation and ferrous ironcatalysed ozonation. J. Hazard, 85(3): 229-24. DOI: $\quad 10.1016 / \mathrm{s} 0304-$ 3894(01)00232-1

Azimi AA, Zamanzadeh M. 2004. Determination of design criteria for UASB reactor as a wastewater pretreatment system in tropical small communities. Inter. J. Environ. Sci. Technol., 1(1): 554-57. DOI : https://doi.org/10.1007/BF03325816.

Bancolé A, Brissaud F, Gnagne T. 2003. Oxidation processes and cloggind in intermittent infiltration. Water SCI. Technol., 48(11-12): 139-146.

Bayoko A. 2015. Caractérisation des eaux usées dans les grands collecteurs du district d'Abidjan. Mémoire de master en ingénierie de l'eau et de l'environnement 2IE (Institut international de l'eau et de l'environnement), Ouagadougou, p. 57.

Colombano S, Saada A, Guerin V, Bataillard P, Bellenfant G, Beranger S, Hube D, Blanc C, Zornig C, Girardeau I. 2010. Quelles techniques pour quels traitementsAnalyse côuts-bénéfices. Rapport final BRGM-RP-58609-FR, p.403.

De Haas DW, Wentzel MC, Ekama GA. 2001. The use of simultaneous chemical precipitation in modified activated sludge systems exhibiting biological excess phosphate removal part 6: modeling of simultaneous chemical-biological $\mathrm{P}$ removal-review of existing models. Water SA., 27(2): 135-150.

Deschamps T, Benzaazoua M, Bussière $\mathrm{B}$, Belem T, Mbonimpa M. 2006. Mécanismes de rétention des métaux lourds en phase solide : cas de la stabilisation des sols contaminés et des déchets industriels. VertigO Rev. Sci. Environ., $\quad 7(2)$ : $1-6$. https://doi.org/10.4000/vertigo.2171

Dovonou F, Aina M, Boukari M, Alassane A. 2011. Pollution physico-chimique et bactériologique d'un écosystème aquatique et ses risques écotoxicologiques: cas du lac Nokoué au 
sud Bénin. Int. J. Biol. Chem. Sci., 5(4): 1590-1602. DOI: 10.4314/ijbcs.v5i4.23.

Edwin L, Declanp P, Paul P, Peter D, Stuart M, John H. 2006. Evaluation of roughing filtration for pre-treatment of storm water prior to Aquifer Storage and Recovery (ASR). CSIRO Land and Water Science Report, ISSN. Australia, 154p.

Effebi KR, Adou KE, Koffi T, Ehouman KS, Goné LD, Perrin JL, Kamagaté B, Dabissi ND, Ségui L. 2017. Assessment of the pollution in Aghien lagoon and its tributaries (Côte d'Ivoire, West Africa). Int. J. Biol. Chem. Sci., 11(1): 515-530. DOI: http://dx.doi.org/10.4314/ijbcs.v11i1.41

El haouti R, Azougarh Y, Et-taleb S, Aba-aaki R, Abbaz M, Lhanafi S, Ez-zahery M, El Alem N. 2016. Traitement de la pollution organique des eaux usées domestiques par le procédé d'infiltration-percolation sur deux types de sables titanifères : brut et tamisé. J. Mater. Environ. Sci., 7(1): 59-66. DOI: 10.13140/RG.2.1.3177.3204

Fouad S, Hajjami K, Cohen N, Chlaida M. 2014. Qualité physico-chimique et contamination métallique des eaux de l'Oued Hassar : impacts des eaux usées de la localité de Mediouna (Périurbain de Casablanca, Maroc). Afr. Sc., 10(1) : 91102.

Georgantas DA, Grigoropoulou HP. 2006. Phosphorus and organic matter removal from synthetic wastewater using alum and aluminum hydroxide. Global NEST Journal, 8(2): 121-130.

Gueye MN. 2007. Contribution à l'étude du traitement des effluents d'un bassin de maturation par filtration sur gravier à Ouagadougou. Mémoire de master, 2IE, Ouagadougou, Burkina Faso, p. 72.

Healy MG, Rodgers M, Mulqueen J. 2007. Performance of stratified filter in removal of chemical oxygen demand, total suspended solids and ammonia nitrogen from high-strength wastewaters. $J$. Environ. Manage., 8: 409-415. DOI: 10.1016/j.jenvman.2006.03.005

Hericher M. 2012. Évaluation de l'impact des dispositifs d'assainissement non collectif sur le sol. Mémoire de Master École
Nationale des Ponts et Chaussées, Paris, p. 57.

Kedi ABB, Kouassi SS, Coulibaly V, Sei J. 2021. Élimination de polluants des déchets liquides d'une unité de production de sucre par des argiles naturelles de Côte d'Ivoire. Int. J. Biol. Chem. Sci., 15(2): 803-815. DOI: https://dx.doi.org/10.4314/ijbcs.v15i2.31

Koanda H. 2006. Vers un assainissement urbain durable en Afrique subsaharienne : approche innovante de planification de la gestion des boues de vidange. Thèse de Doctorat, Ecole Polytechnique Fédérale de Lausanne, Lausanne, Suisse, p. $276+$ Annexes.

Koné DM. 2011. Infiltration-percolation sur sable et sur fibres de coco, filtres plantes et épuration des eaux usées domestiques à dominance agroalimentaire sous climat tropical sec/ cas des eaux résiduaires de Ouagadougou, Thèse de doctorat, université de Ouagadougou, Ouagadougou, p. 232.

Krou NJ. 2010. Etude expérimentale et modélisation d'un procédé séquentiel AD-OX d'élimination de polluants organiques. Thèse de Doctorat, Université de Toulouse, Toulouse, p. 208.

Le Cloirec P. 2004. COV (composés organiques volatils). Techniques de l'Ingénieur G 1 835: 1-10.

Mangoua Allali ALC, Coulibaly L. 2015. Filtres à sable immerge à alimentation intermittente : effet de la hauteur de sable sur l'élimination des paramètres physicochimiques. IJIAS, 11(2): 418-428.

Massé DI, Masse L. 2000. characterization of wastewater from hog slaughterhouses in Earstern Canada and evaluation of their in-plant wastewater treatment systems. Can. Agric. Engin., 42(3): 139-146.

Meçabih Z, Kacimi S, Bouchikhi B. 2006. Adsorption des matières organiques des eaux usées urbaines sur la bentonite modifiée par $\mathrm{Fe}(\mathrm{III}), \mathrm{Al}(\mathrm{III})$ et $\mathrm{Cu}(\mathrm{II})$. Rev. Des Sci. De l'Eau / J. Water Sci., 19(1) : 23-31. DOI : https://doi.org/10.7202/012261ar

Monette F, Brière FG, Létourneau M, Duchesne M, Hausler R. 2000. Traitement des eaux usées par 
coagulation-floculation avec recirculation des boues chimiques : Performance générale et stabilité du procédé. Can. J. Civ. Eng., 27: 702-718. DOI: $10.1139 /$ cjce-27-4-735

Ménoret C. 2001. Traitement d'effluents concentrés par cultures fixées sur gravier ou pouzzolane. Thèse de doctorat Université Montpellier II, Montpellier, p. $124+$ annexes.

Ministère de l'Environnement, des Eaux et forêts $2008 . \quad$ Arrêté $\mathrm{N}^{\circ}$ 01164/MINEEF/CIAPOL/SDIIC du 04 Nov. 2008 portant Réglementation des Rejets et Emissions des Installations Classées pour la Protection de l'Environnement. Ministère de l'Environnement, des Eaux et forêts/Centre Ivoirien Antipollution, Côte d'Ivoire.

Mosely J. 2001. Enhanced CBOD and nitrogen removal in recirculating sand filter wastewater treatment system by addition of plastic media to the recirculating tank. Sumner \& Cannon, Inc. Nashville, Tennessee, USA. 48 p.

Moukorab AMD, Aïna MP, Agoungbome D. 2018. Élimination de la matière organique par le procédé d'électrocoagulation : comparaison à la coagulation chimique. Déchets Sciences et Techniques, (72): 17. DOI: $10.4267 /$ dechets-sciencestechniques.3489

Ouattara PJM. 2005. Fonctionnement des grands collecteurs d'eaux pluviales dans cinq communes d'Abidjan-Nord (Abobo -Attécoubé-Adjamé-Cocody et Yopougon). Mémoire de DEA, Université d'Abobo-Adjamé, Abidjan, p.66.

Ouattara J-MP, Coulibaly L. 2019. Effet de la charge hydraulique appliquée sur le fonctionnement d'un marais artificiel à drainage vertical planté avec Panicum maximum traitant des eaux domestiques. Int. J. Biol. Chem. Sci., 13(5): 24-38. DOI: https://dx.doi.org/10.4314/ijbcs.v13i5.2S

Renou S. 2006. Analyse de cycle de vie appliquée aux systèmes de traitement des eaux usées. Thèse de Doctorat, Institut
National Polytechnique de Lorraine, Lorraine, p. 260.

Salghi R. 2007. Différentes filières de traitement des eaux Université IBN ZOHR. ENSA, 8 p.

Shaimali M, Singh KP. 2001. New methods of nitrate removal from water. Environ. Poll., 112: 351-359. DOI: 10.1016/s0269-7491(00)00147-0

Taghizadeh MM, Torabian A, Borghei M, Hassani AH. 2007. Feasibility study of water purification using vertical porous concrete filter. Int. J. Environ. Sci. Tech., 4(4): 505-512.

Tanja R-W, Jörg ED. 2006. Using soil biomass as an indicator for the biological removal of effluent-derived organic carbon during soil infiltration. Wat. Res., 40: 961-968. DOI: 10.1016/j.watres.2006.01.007

Tatangelo A. 2006. Optimisation de la précipitation des métaux lourd en mélange et en valorisation des boues d'hydroxydes : application aux effluents de traitement de surfaces. Thèse de Doctorat, École Nationale supérieure des mines Saint Ettienne, France, p. 296.

Tchobanoglous G, Burton FL, Stensel HD. 2003. Wastewater Engineering: Treatment and Reuse (4th Edn). McGraw-Hill: New York; p.213.

Van Kaam R. 2005. Bioréacteur à membranes immergées pour le traitement d'eaux usées domestiques, influence des conditions d'infiltration et de l'hydrodynamique sur les performances du procédé. Thèse de Doctorat, Université de Toulouse, Toulouse, p. 213.

Yu J, Chen H, Ji M, Yue PL. 2000. Distribution and change of microbial activity in combined UASB and AFB reactor of wastewater treatment. Bioprocess Engineering, 22: 315-322. 